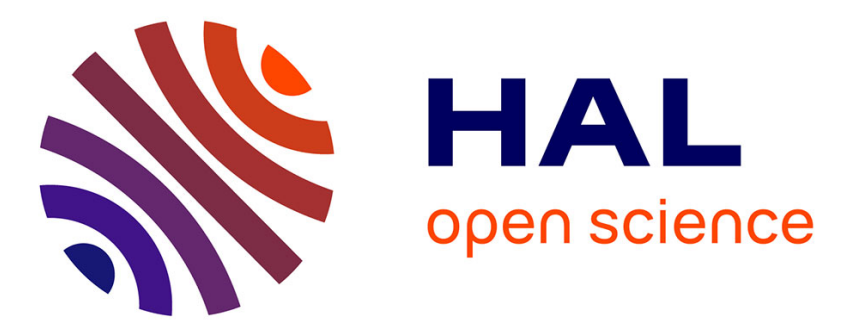

\title{
Relations entre les pucerons des céréales et leurs plantes-hôtes. I. - Mise au point d'une méthodologie de recherche de résistance à Sitobion avenae (F.) chez différents cultivars de blé d'hiver
}

\author{
Jean-Paul Di Pietro, Charles-Antoine Dedryver, Christine Soster
}

\section{To cite this version:}

Jean-Paul Di Pietro, Charles-Antoine Dedryver, Christine Soster. Relations entre les pucerons des céréales et leurs plantes-hôtes. I. - Mise au point d'une méthodologie de recherche de résistance à Sitobion avenae (F.) chez différents cultivars de blé d'hiver. Agronomie, 1986, 6 (5), pp.468-479. hal-00884899

\section{HAL Id: hal-00884899 \\ https://hal.science/hal-00884899}

Submitted on 1 Jan 1986

HAL is a multi-disciplinary open access archive for the deposit and dissemination of scientific research documents, whether they are published or not. The documents may come from teaching and research institutions in France or abroad, or from public or private research centers.
L'archive ouverte pluridisciplinaire HAL, est destinée au dépôt et à la diffusion de documents scientifiques de niveau recherche, publiés ou non, émanant des établissements d'enseignement et de recherche français ou étrangers, des laboratoires publics ou privés. 


\section{Relations entre les pucerons des céréales et leurs plantes-hôtes. I. - Mise au point d'une métho- dologie de recherche de résistance à Sitobion avenae (F.) chez différents cultivars de blé d'hiver}

Jean-Paul DI PIETRO \& Charles-Antoine DEDRYVER avec la collaboration technique de Christine SOSTER

I.N.R.A., Laboratoire de Recherches de la Chaire de Zoologie de l'E.N.S.A.R., Centre de Recherches de Rennes, F 35650 Le Rheu

La recherche de relations d'antibiosis entre Sitobion avenae (F.) et différents cultivars de blé d'hiver a été effectuée en caractérisant chaque cultivar par le taux intrinsèque d'accroissement journalier $\left(\mathrm{r}_{\mathrm{m}}\right)$ qu'exprime ce puceron en conditions contrôlées et à 2 stades phénologiques : plantules non vernalisées et épiaison-maturité laiteuse.

Vingt-cinq cultivars ont été étudiés dont 18 choisis par sélection au champ dans une collection de blés d'hiver. Ceci nous a permis de classer, pour chaque stade phénologique étudié, les cultivars en 3 groupes (fortement, moyennement et peu multiplicateurs de $S$. avenae) et de montrer que :

- pour la plupart des cultivars, le $r_{\mathrm{m}}$ est plus élevé au stade épiaison qu'au stade plantule,

- selon le stade phénologique étudié, certains cultivars peuvent appartenir à des groupes d'infestation différents.

Nous avons mis en évidence que " Maris Huntsman " et "Champlein " sont, dans les 2 situations, fortement multiplicateurs de $S$. avenae alors que « Cajka » et «Vala » manifestent une résistance partielle à ce puceron. D'autres cultivars («Iljitchevka », " Nongda 140 », " Jubilejna » et «PI 94571 ») présentent à l'épiaison un caractère peu multiplicateur de $S$. avenae également remarquable.

La méthodologie et l'intérêt de la détermination du $r_{m}$ sont discutés.

Mots clés additionnels : Aphides, Triticum aestivum, effet variétal, taux intrinsèque d'accroissement. identifying resistance to Sitobion avenae $(F$.$) by antiobiosis in winter wheat.$

The search for antibiosis relations between Sitobion avenae (F.) and different cultivars of winter wheat was carried out by characterizing each cultivar by the daily intrinsic rate of increase $\left(\mathrm{r}_{\mathrm{m}}\right)$ of the aphid in a controlled environment at 2 growth stages of the plant : non-vernalized plant and heading-milky ripeness. Twenty five cultivars were studied, including 18 chosen by field selection in a winter wheat collection. The results allowed cultivars to be classified, at each growth stage, into 3 groups depending on their ability to multiply $S$. avenae (strongly, moderately, poorly). For most of the cultivars, $\mathrm{r}_{\mathrm{m}}$ was higher at heading than at the non-vernalized plant stage. Some cultivars belonged to different infestation groups at different growth stages. "Maris Huntsman" and "Champlein" multiplied $S$. avenae strongly at both stages, while "Cajka" and "Vala" were partially resistant to this aphid. Other cultivars ("Iljitchevka", "Nongda 140", "Jubilejna" and "PI 94571") multiplied $S$. avenae poorly at heading, which is remarkable. The methodology and the value of assessment of $r_{m}$ were discussed.

Additional key words : Aphids, Triticum aestivum, varietal effects, intrinsic rate of increase. 


\section{INTRODUCTION}

L'un des objectifs essentiels de la lutte intégrée consiste à essayer de maintenir la densité numérique des animaux nuisibles au-dessous d'un seuil déterminé, à partir duquel les déprédations de ces ravageurs risquent d'avoir une incidence économique non négligeable sur la production. Le choix comme moyen préventif de variétés d'espèces cultivées relativement peu favorables à la multiplication de leurs déprédateurs peut contribuer de manière essentielle à atteindre ce but, surtout si ces animaux présentent une vitesse de développement et un taux de reproduction élevés.

Tel est le cas en particulier de Sitobion avenae (F.), le puceron des épis des céréales, reconnu depuis une quinzaine d'années seulement comme la principale espèce de pucerons responsable de dégâts directs sur blé en Europe du Nord (VEREIJKEN, 1979). L'intérêt d'une telle approche se trouve justifié historiquement par la constatation de LOWE (1982) qui propose comme possible explication de l'accroissement récent des problèmes posés par ce puceron en GrandeBretagne, le remplacement, vers 1970 , de variétés de blé d'hiver, telle "Capelle Desprez " qu'il juge relativement " résistante " au puceron, par d'autres plus récentes comme « Joss Cambier » ou " Maris Huntsman " décrites comme très favorables à ce puceron.

Dans ce contexte, un de nos objectifs est de prospecter une gamme de cultivars aussi large que possible afin de déceler, chez certains d'entre eux, d'éventuels degrés de "résistance " susceptible d'être introduite dans un programme d'amélioration génétique. Un autre objectif sera d'établir un classement des principales variétés de blé actuelles en fonction de leur caractère multiplicateur de $S$. avenae et ultérieurement d'autres pucerons des céréales, permettant d'attribuer à chaque variété, lorsqu'elle est cultivée, un plus ou moins grand risque de pullulation de ces ravageurs.

Ces phénomènes de résistance relative sont complexes dans leurs mécanismes : PAINTER $(1951,1958)$ distingue la non-préférence (redéfinie sous le terme d'antixénosis par KOGAN \& ORTMAN, 1978), l'antibiosis et la tolérance. Aucune étude n'a encore porté sur la tolérance, c'est-à-dire sur la capacité qu'ont certains cultivars de se développer en présentant finalement des dégâts acceptables en dépit de niveaux de populations du ravageur qui provoqueraient des dommages importants sur d'autres hôtes sensibles. Pour ce qui est des phénomènes d'antixénosis et d'antibiosis qui affecteraient, selon CARTER \& DIXON (1981), respectivement le taux d'immigration des pucerons sur la plante-hôte et leurs paramètres biotiques (durée de développement, fécondité, taux de survie, ...), les modalités expérimentales permettent difficilement de les mettre en évidence de manière isolée. Les observations de DEDRYVER \& DI PIETRO (1986) en conditions naturelles montrent que ces phénomènes peuvent être masqués ou modifiés par l'action d'autres facteurs intrinsèques (précocité des variétés, sensibilité aux maladies cryptogamiques, ...). En serre, où les pucerons peuvent circuler d'un cultivar à l'autre (« the walk-about method ») et où la résistance est appréciée en dénombrant les populations de $S$. avenae sur chaque cultivar un certain temps après l'infestation initiale par des pucerons immatures, les travaux de LowE $(1980,1981,1982)$ ne permettent pas davantage de distinguer l'effet d'antixénosis de l'antibiosis. C'est pourquoi, de la même manière que SOTHERTON \& VAN EMDEN (1982), nous avons entrepris, en 1981, des expérimentations en conditions contrôlées qui permettent d'étudier séparément chacun des 2 phénomènes et donc de définir avec précision l'éventuel facteur de résistance à sélectionner (DI PIETRO \& DEDRYVER, 1984).

Nous avons choisi d'étudier en premier lieu l'effet d'antibiosis. Celui-ci est mesuré par l'action dépressive que peut exercer le cultivar hôte sur l'expression des différentes composantes de l'accroissement des populations de $S$. avenae : durée de développement e! mortalité larvaire, taux de survie et fécondité journalière des virginipares. C'est ce qu'intègre la détermination du taux intrinsèque d'accroissement naturel tel qu'il est défini par BIRCH (1948). Ce paramètre, estimé pour des individus de $S$. avenae élevés sur différents cultivars dans des conditions identiques, peut s'interpréter comme un indice, propre à chaque cultivar, de capacité à favoriser ou défavoriser la multiplication de ce puceron.

\section{MATÉRIEL ET MÉTHODE}

\section{A. Les cultivars de blé d'hiver}

Vingt-cinq cultivars au total ont fait l'objet d'études. Sept d'entre eux ont été retenus parmi ceux étudiés par DEDRYvER \& Di PIETRo $(1984,1986)$ en conditions naturelles. Il s'agit :

- de variétés très cultivées en France, surtout lors des années 1977-1978, et très infestées au champ telle «Maris Huntsman », ou présentant des niveaux variables d'infestation selon les années comme « Capitole ", " Champlein ", " Fidel " et " Roazon ";

- de lignées signalées comme peu favorables aux pucerons telle la lignée russe "Mironovskaïa 808 " (HINZ \& DAEBELER, 1974, 1976) ou la lignée « $(\mathrm{Mi} \times \mathrm{b})$ 5-4-4-3-2 ", issue de "Mironovskaïa » et "Talent ", considérée à la suite de travaux préliminaires comme peu multiplicatrice pour S. avenae (DI PIETRO \& DEDRYVER, 1984).

Les abréviations utilisées pour ces cultivars dans les tableaux et figures sont respectivement: MHUN, CAPI, CHAM, FIDE, ROAZ, MIR8 et MIXB.

Dix-huit autres cultivars ont été choisis à partir d'observations effectuées au champ sur une collection de 212 lignées de blé d'hiver de la Station d'Amélioration des plantes de l'I.N.R.A. du Rheu, en juin 1983. Ces cultivars se trouvaient alors à un stade phénologique variant entre l'émergence des épis et la maturité laiteuse: stades 50 à 75 de l'échelle de ZADOKS (ZADOKS et al., 1977). Chaque cultivar occupait une placette de 4 rangées de $2 \mathrm{~m}$ de long. Sur 20 talles choisies au hasard le long des 2 rangées centrales, un dénombrement des individus isolés et des colonies de différentes tailles a été effectué pour $S$. avenae. En même temps, on a noté les effectifs globaux de Rhopalosiphum padi (L.) et de Metopolophium dirhodum (Wlk.), moins nombreux à cette époque. Les effectifs moyens sur 20 talles de l'ensemble de la collection et le critère de choix de cultivars supposés peu favorables principalement à $S$. avenae sont présentés dans le tableau 1. Le tableau 2 donne la liste des cultivars 


\section{TABLEAU 1}

Effectifs moyens de $\mathrm{S}$. avenae observés dans une collection de 212 cultivars de blé d'hiver

et effectifs limites retenus pour le choix de lignées apparemment peu favorables à ce puceron.

Mean populations of $\mathrm{S}$. avenae observed in a collection of 212 cultivars of winter wheat and limit populations for the choice of obviously poorly favourable lines to this aphid.

\begin{tabular}{|c|c|c|c|}
\hline & & $\begin{array}{l}\text { Effectifs moyens } \\
\text { de la collection } \\
\text { (pour } 20 \text { talles) }\end{array}$ & $\begin{array}{c}\text { Effectifs limites } \\
\text { retenus } \\
\text { (pour } 20 \text { talles) }\end{array}$ \\
\hline \multirow{6}{*}{ 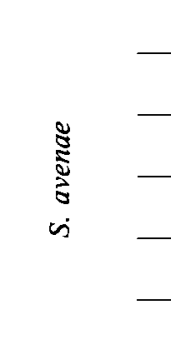 } & Nombre d'individus isolés & 7,94 individus & 4 individus \\
\hline & $\mathrm{Nb}$. de colonies de 2 à 5 ind. & 6,13 colonies & 3 colonies \\
\hline & Nb. de colonies de 6 à 10 ind. & 1,84 colonies & 0 colonie \\
\hline & $\mathrm{Nb}$. de colonies de 11 à 20 ind. & 0,48 colonies & 0 colonie \\
\hline & $\mathrm{Nb}$. de colonies plus de 20 ind. & 0,18 colonies & 0 colonie \\
\hline & Effectif moyen estimé & 57 individus & 14 individus \\
\hline R. padi & Effectif moyen & 6,89 individus & 3 individus \\
\hline M. dirhodum & Effectif moyen & 2,85 individus & 1 individu \\
\hline
\end{tabular}

TABLEAU 2

Lignées apparemment peu favorables à $\mathrm{S}$. avenae retenues parmi 212 cultivars de blé d'hiver de la collection de la Station I.N.R.A. d'Amélioration des Plantes, Le Rheu.

Obviously poorly favourable lines to S. avenae chosen among 212 cultivars of winter wheat of the collection of the Plant Breeding Station, I.N.R.A., Le Rheu.

\begin{tabular}{|c|c|c|c|c|c|c|c|}
\hline & & & & & tifs sur 20 & & \\
\hline & & & & Sitobion avenae & & R. padi & M. dirhodum \\
\hline & Lignées & $\begin{array}{l}\text { Nom } \\
\text { abrégé }\end{array}$ & Nombre & Nombre & Effectif & Effectif & Effectif \\
\hline 8 & « Campeneac $3 »$ & (CAM3) & 1 & 0 & 1 & 0 & 0 \\
\hline 9 & «Campeneac $7 »$ & (CAM7) & 4 & 1 & 7,5 & 0 & 0 \\
\hline 10 & "Campeneac $13 »$ & (CA13) & 3 & 0 & 3 & 0 & 0 \\
\hline 11 & «Cadet» & $(\mathrm{CADE})$ & 4 & 1 & 7,5 & 0 & 0 \\
\hline & "Cauel" & & 7 & 1 & 10,5 & 0 & 0 \\
\hline 12 & «Blé rouge d'hiver barbu » & (BRHB) & 2 & 3 & 12,5 & 33 & 0 \\
\hline 13 & «Achtyrcanka » & $(\mathrm{ACHT})$ & 7 & 2 & 14 & 4 & 0 \\
\hline 14 & «Cajka » & (CAJK) & 4 & 3 & 14,5 & 0 & 0 \\
\hline 15 & "Jubilejna " & (JUBI) & 4 & 0 & 4 & 2 & 0 \\
\hline 16 & «Mironovskaïa-Nizkorostla » & $(\mathrm{NIZK})$ & 3 & 3 & 13,5 & 1 & 0 \\
\hline 17 & «Mironovskaïa X Ouloutchennaïa » & (MIXO) & 3 & 2 & 10 & 0 & 1 \\
\hline & & & 11 & 1 & 4,5 & 0 & 0 \\
\hline 18 & «lliitchevka» & (II JI) & $\int 2$ & 0 & 2 & 0 & 3 \\
\hline & " ijitchevka 》 & & 9 & 0 & 9 & 0 & 0 \\
\hline 19 & «Mironoyskaïa $25 »$ & (MI25) & $\int 1$ & 1 & 4,5 & 0 & 0 \\
\hline & & & $\{10$ & 2 & 17 & 1 & 0 \\
\hline- & "Mironovskaïa $808 "$ & (MIR8) & 2 & I & 5,5 & 1 & 0 \\
\hline & & & 7 & 1 & 10,5 & 0 & 0 \\
\hline 20 & «Kianka » & (KIAN) & 3 & 3 & 13,5 & 1 & 0 \\
\hline & & & 7 & 1 & 10,5 & 3 & 0 \\
\hline 21 & «Vala » & (VALA) & 7 & 1 & 10,5 & 34 & 0 \\
\hline 22 & « Nongda $140 »$ & (ND40) & 2 & 0 & 2 & 1 & 0 \\
\hline 23 & « Nongda $141 »$ & (ND41) & 6 & 0 & 6 & 1 & 0 \\
\hline 24 & «PI $94585 »$ & (PI85) & 1 & 3 & 11,5 & 0 & 0 \\
\hline 25 & «PI $94571 »$ & (PI71) & 8 & 0 & 8 & 3 & 0 \\
\hline & "Canitole & (CAPD) & 11 & 2 & 18 & 0 & 0 \\
\hline & 《Capitole » & (CAPI) & $\{16$ & 10 & 120 & 5 & 0 \\
\hline Effect & ffs limites pour la sélection & & 4 & 3 & 14 & 3 & 1 \\
\hline
\end{tabular}


ainsi retenus, la nomenclature abrégée utilisée dans les tableaux et figures et les effectifs relevés sur chacun d'entre eux. La lignée "Blé rouge d'hiver barbu » a été retenue, malgré d'importantes infestations par $R$. padi, pour ses épis dont la forte aristation peut avoir une influence sur l'installation de $S$. avenae. Les cultivars 13 à 21 sont originaires d'U.R.S.S. (Ukraine). On y retrouve "Mironovskaïa 808 » déjà cité plus haut. Le cultivar «Vala » a également été retenu car il est issu d'un croisement entre "Mironovskaïa » et «Moisson ». Les cultivars 22 et 23 sont d'origine chinoise (Université de Pékin). Enfin les cultivars 24 et 25 sont respectivement d'origine portugaise et grecque.

La variété "Capitole ", présente dans cette collection, portait des effectifs moyens de pucerons supérieurs à ceux retenus comme critère de choix de cultivars peu favorables.

\section{B. Le matériel animal : Sitobion avenae}

A partir d'une souche maintenue en serre à l'E.N.S.A. de Rennes depuis 1980 sur la variété «Champlein », des élevages de $S$. avenae sont réalisés sur plantes vernalisées de chaque cultivar à $16 \pm 1,5^{\circ} \mathrm{C}$ et $16 \mathrm{~h}$ de photophase. Ils sont renouvelés avant surpopulation tous les $20 \mathrm{j}$ environ. A partir de la $3^{\mathrm{e}}$ génération au moins, obtenue sur un cultivar donné, des larves aptères du $4^{\mathrm{e}}$ stade peuvent être prélevées : ce sont les premières larves issues de ces individus parvenus au stade virginipare aptère qui sont utilisées dans les expériences concernant ce même cultivar.

\section{Méthode expérimentale}

\section{Modalités d'expérience}

Les expériences ont été menées dans 2 chambres climatisées maintenues à $20 \pm 1,5^{\circ} \mathrm{C}, 16 \mathrm{~h}$ de photophase et une humidité relative de $50-75$ p. 100 , à 2 stades phénologiques de la plante-hôte.

\section{a) Stade jeune plantule non vernalisée}

Pour chacun des 25 cultivars, des semences sont placées sur vermiculite dans des tubes de $12 \mathrm{~cm}$ de hauteur et $2,5 \mathrm{~cm}$ de diamètre. Des plantules de 2 à 3 feuilles (stade 12-13 de l'échelle de ZADOKS) sont obtenues au bout de $7 \mathrm{j}$. Elles sont arrosées une fois par semaine avec une solution nutritive équilibrée $(6 \mathrm{~N}-3 \mathrm{P}-5 \mathrm{~K})$.

\section{b) Stade épiaison}

D'autres graines sont semées en pots de $12 \times 12 \mathrm{~cm}$ dans un mélange de terre franche, terreau et sable. Après germination, les plantules sont placées en conditions de vernalisation $\left(6 \pm 2{ }^{\circ} \mathrm{C} ; 12 \mathrm{~h}\right.$ de photophase) pendant au moins 8 semaines, puis mises en conditions naturelles. A l'émergence des épis (stade 59 de l'échelle de ZADOKS), les plantes sont installées en chambre climatisée. La lignée «Campénéac 13 » n'a pu être étudiée à ce stade.

Une larve néonate est installée selon le cas sur chaque plantule ou épi, isolés dans un sachet de cellophane d'environ $15 \mathrm{~cm}$ de long et $2,5 \mathrm{~cm}$ de diamètre, perméable à l'air et permettant une parfaite visibilité.

\section{Les données recueillies}

Les observations quotidiennes permettent de noter la mortalité et la durée de développement des larves. En général, une forte proportion de virginipares aptères est obtenue pour laquelle on note la durée qui précède la ponte des premières larves, la fécondité journalière et la longévité. Tous les $7 \mathrm{j}$ environ, on procède au retrait de leur descendance afin d'éviter un mélange des générations. En moyenne, 11 répétitions ont pu être retenues pour chaque cultivar au stade plantule, après élimination des individus dont la longévité était inférieure à $15 \mathrm{j}$ : cette mortalité «précoce " assez élevée (30 à 50 p. 100 selon les cultivars) peut être, en grande partie, attribuée plus vraisemblablement à des plantules déficientes ou aux manipulations effectuées qu'à un effet d'antibiosis. Pour les mêmes raisons, on n'a pas pris en compte la mortalité aux stades larvaires d'ailleurs très faible (environ 3 p. 100). Au stade épiaison, on a retenu, en moyenne, 8 répétitions par cultivar : à ce stade, la mortalité larvaire est nulle et celle intervenant avant $10 \mathrm{j}$ de vie imaginale est très faible $(0,5 \mathrm{p}$. 100).

\section{Le traitement des données}

Les données ainsi recueillies permettent de calculer la valeur du taux intrinsèque d'accroissement journalier $\left(r_{m}\right)$ exprimé par $S$. avenae sur chaque cultivarhôte étudié, dans nos conditions d'expérience : ce paramètre est défini par BIRCH (1948) comme le taux d'accroissement par individu, dans des conditions abiotiques et nutritionnelles déterminées et dans un milieu où les effets d'augmentation de la densité ne se font pas sentir. Il présente un grand intérêt en tant qu'indice de comparaison entre différentes conditions, soit, dans notre cas le caractère favorable ou défavorable, à un stade phénologique donné, des diffétents cultivars pouvant agir sur la vitesse de multiplication d'une population de $S$. avenae.

Un programme FORTRAN 77, adapté d'un programme ALGOL 68 mis au point au Département de Biométrie de l'Université de Southampton (GrandeBretagne) permet de calculer le $\mathrm{r}_{\mathrm{m}}$ de $S$. avenae qui caractérise chaque cultivar-hôte, son écart-type et sa variance, estimés à partir des données propres à chaque répétition par la méthode de QUENOUILLETUCKEY (ou "méthode de Jacknife ") exposée par LEBART et al. (1979).

\section{RÉSULTATS}

\section{A. Les essais au stade plantule non vernalisée}

Le tableau 3 présente les valeurs moyennes des paramètres mesurés à ce stade et du $\mathrm{r}_{\mathrm{m}}$ de $S$. avenae sur les 25 cultivars par ordre décroissant de $r_{m}$.

Deux types d'analyses statistiques ont été effectuées afin, d'une part, de comparer l'intérêt du paramètre synthétique qu'est le $r_{m}$ à celui des différents paramètres relevés et, d'autre part, de classer les cultivars étudiés en groupes favorables ou défavorables à la multiplication de $S$. avenae.

Une analyse en composantes principales (A.C.P.) a 
TABLFAU 3

Caractéristiques démographiques de $\mathrm{S}$. avenae sur 25 cultivars de blé d'hiver au stade plantule non vernalisée. Les écarts-types des valeurs moyennes sont indiqués entre parenthèses. Les $r_{m}$ ayant une lettre en commun ne sont pas significativement différents au seuil 1 \% du test de DuNCAN. Demographic characteristics of S. avenae on 25 cultivars of winter wheat at the "non-vernalized plant " stage. Standard deviations of the means are between brackets. The $r_{m}$ with a common letter did not differ significantly at the I \%o threshold of DuNCAN's range test.

\begin{tabular}{|c|c|c|c|c|c|c|c|c|c|c|c|}
\hline \multirow{2}{*}{$\begin{array}{c}\text { Cultivars } \\
\text { Pl85 }\end{array}$} & \multirow{2}{*}{$\begin{array}{l}(1) \\
08\end{array}$} & \multicolumn{2}{|c|}{$\begin{array}{l}\text { Age reproducteur } \\
\text { (jour) }\end{array}$} & \multicolumn{2}{|c|}{$\begin{array}{l}\text { Nombre de jours } \\
\text { de ponte }\end{array}$} & \multicolumn{2}{|c|}{$\begin{array}{l}\text { Fécondité totale } \\
\text { (Larves/Q) }\end{array}$} & \multicolumn{2}{|c|}{$\begin{array}{c}\text { Fécondité journalière } \\
\text { (Larves/ Q / jour) }\end{array}$} & \multicolumn{2}{|c|}{$\begin{array}{l}\text { Taux intrinsèque } \\
\text { d'accroissement } \\
\text { journalier } r_{m}\end{array}$} \\
\hline & & 8,13 & $(0,35)$ & 27,63 & $(3,11)$ & 63,38 & $(09,75)$ & 2,38 & $(0,55)$ & 0,2936 & $(0,025) \quad a$ \\
\hline CADE & 13 & 8,23 & $(0,44)$ & 30,23 & $(6,56)$ & 78,31 & $(08,83)$ & 2,69 & $(0,58)$ & 0,2929 & $(0,020) \quad \mathrm{a}$ \\
\hline CHAM & 07 & 8,43 & $(0,53)$ & 22,00 & $(7,30)$ & 67,00 & $(21,20)$ & 3,07 & $(0,36)$ & 0,2915 & $(0,021) \quad a$ \\
\hline ILJI & 14 & 8,57 & $(0,65)$ & 27,07 & $(6,84)$ & 67,64 & $(08,67)$ & 2,63 & $(0,65)$ & 0,2913 & $(0,027)$ a \\
\hline MHUN & 13 & 8,54 & $(0,52)$ & 27,77 & $(7,60)$ & 70,31 & $(10,77)$ & 2,69 & $(0,69)$ & 0,2896 & $(0,020) \quad a$ \\
\hline FIDE & 13 & 8,31 & $(0,48)$ & 28,08 & $(7,05)$ & 65,62 & $(16,50)$ & 2,42 & $(0,63)$ & 0,2868 & $(0,022)\{a b$ \\
\hline ND41 & 10 & 8,40 & $(0,70)$ & 28,50 & $(5,62)$ & 65,40 & $(12,44)$ & 2,33 & $(0,42)$ & 0,2858 & $(0,030)\{a b c$ \\
\hline CAM3 & 11 & 8,36 & $(0,50)$ & 23,55 & $(5,63)$ & 63,45 & $(16,38)$ & 2,70 & $(0,49)$ & 0,2825 & $(0,026)\{\mathrm{abcd}$ \\
\hline PI71 & 09 & 8,67 & $(1,32)$ & 25,56 & $(5,13)$ & 60,44 & $(06,25)$ & 2,43 & $(0,44)$ & 0,2821 & $(0,029)\{a b c d$ \\
\hline $\mathrm{CAM} 7$ & 14 & 8,57 & $(0,51)$ & 29,14 & $(8,20)$ & 68,07 & $(17,87)$ & 2,41 & $(0,50)$ & 0,2791 & $(0,015)$ \{abcd \\
\hline JUBI & 15 & 8,60 & $(0,51)$ & 26,87 & $(3,89)$ & 66,67 & $(09,73)$ & 2,57 & $(0,29)$ & 0,2784 & $(0,019)$ \{abcd \\
\hline ROAZ & 11 & 8,45 & $(0,69)$ & 29,64 & $(7,18)$ & 62,00 & $(14,95)$ & 2,12 & $(0,28)$ & 0,2769 & $(0,026)\{a b c d$ \\
\hline MI25 & 09 & 9,00 & $(0,71)$ & 25,78 & $(7,55)$ & 60,33 & $(07,57)$ & 2,53 & $(0,81)$ & 0,2755 & $(0,032)$ labcd \\
\hline CAPI & 16 & 8,56 & $(0,81)$ & 27,88 & $(7,14)$ & 62,50 & $(07,66)$ & 2,41 & $(0,75)$ & 0,2750 & $(0,027)\{\mathrm{abcd}$ \\
\hline CA13 & 14 & 8,64 & $(0,93)$ & 26,86 & $(4,96)$ & 71,36 & $(15,52)$ & 2,66 & $(0,42)$ & 0,2742 & $(0,028)$ \{abcd \\
\hline KIAN & 10 & 8,60 & $(0,52)$ & 22,60 & $(7,81)$ & 60,10 & $(13,28)$ & 2,82 & $(0,71)$ & 0,2741 & $(0,024)$ \{abcd \\
\hline MIXB & 07 & 8,86 & $(0,38)$ & 30,00 & $(4,97)$ & 65,29 & $(08,67)$ & 2,21 & $(0,32)$ & 0,2697 & $(0,020)$ labcd \\
\hline ND40 & 06 & 8,83 & $(0,41)$ & 29,00 & $(4,10)$ & 63,00 & $(16,31)$ & 2,17 & $(0,48)$ & 0,2675 & $(0,013)\{\mathrm{abcd}$ \\
\hline NIZK & 09 & 8,89 & $(0,60)$ & 29,00 & $(6,30)$ & 61,67 & $(08,87)$ & 2,21 & $(0,47)$ & 0,2666 & $(0,012)\{a b c d$ \\
\hline MIXO & 09 & 8,89 & $(1,05)$ & 28,89 & $(6,05)$ & 61,44 & $(11,48)$ & 2,16 & $(0,29)$ & 0,2640 & $(0,038)$ labcd \\
\hline BRHB & 08 & 9,00 & $(0,53)$ & 28,50 & $(5,48)$ & 61,38 & $(10,99)$ & 2,19 & $(0,42)$ & 0,2637 & $(0,021)$ labcd \\
\hline $\mathrm{ACHT}$ & 09 & 9,00 & $(0,71)$ & 30,22 & $(4,71)$ & 64,33 & $(12,78)$ & 2,16 & $(0,44)$ & 0,2625 & $(0,024)$ labcd \\
\hline MIR8 & 15 & 8,87 & $(0,92)$ & 26,00 & $(7,86)$ & 55,60 & $(13,49)$ & 2,20 & $(0,41)$ & 0,255 & $(0,020)$; bcd \\
\hline CAJK & 11 & 9,36 & $(1,63)$ & 29,45 & $(6,83)$ & 61,64 & $(20,78)$ & 2,08 & $(0,62)$ & 0,2528 & $(0,035)$ icd \\
\hline VALA & 11 & 9,27 & $(1,35)$ & 30,82 & $(5,56)$ & 57,82 & $(09,54)$ & 1,96 & $(0,24)$ & 0,2508 & $(0,037) d$ \\
\hline
\end{tabular}

(1) Nombre de répétitions.

porté sur les 5 variables suivantes mesurées pour l'ensemble des individus (272) :

- âge à la reproduction (durée de développement de la naissance au début de la ponte) de chaque individu de $S$. avenae pour un cultivar donné : AGER ;

- nombre de jours de ponte : NJPO ;

- fécondité totale : FECT ;

— fécondité moyenne journalière : FECJ ; RM.

- taux intrinsèque d'accroissement journalier :

Les données sont très bien résumées par les 2 premières composantes qui représentent 78 p. 100 de l'inertie totale. La $1^{\text {re }}(45 \mathrm{p} .100$ de l'inertie $)$ est très fortement corrélée à la variable $\mathrm{RM}$ et, dans une moindre mesure, à AGER (coefficients de corrélation respectifs : 0,874 et 0,543 ). Ces 2 variables sont de plus liées par une forte corrélation négative de coefficient $-0,73$. La $2^{\text {e }}$ composante (33 p. 100 de l'inertie) est principalement corrélée à la variable NJPO (coefficient : 0,975). Les autres variables étudiées, FECT et FECJ, contribuent presque également à la construction de ces 2 axes (coefficients de corrélation compris entre 0,428 et 0,244 ) et sont d'ailleurs également corrélées aux variables RM et NJPO : en valeur absolue les coefficients de corrélation sont compris entre 0,59 et 0,46 .

La figure 1 représente la position des variables et des individus correspondant aux cultivars les plus remarquables sur le plan factoriel défini par les 2 pre- miers axes. Le $1^{\text {er }}$ axe, défini principalement par RM et AGER, peut s'interpréter comme l'expression de la vitesse de multiplication de $S$. avenae : il oppose les cultivars sur lesquels ce puceron se multiplie rapidement (âge à la reproduction court et taux d'accroissement élevé) à ceux sur lesquels il se multiplie lentement. Le $2^{\mathrm{e}}$ axe, défini par NJPO, oppose, indépendamment des cultivars, les individus à longue durée de ponte et grande longévité à ceux ayant une longévité brève : en effet, les virginipares aptères de $S$. avenae ont tendance à échelonner leurs pontes pendant toute leur durée de vie.

En conséquence, on peut donc affirmer qu'à ce stade, le taux intrinsèque d'accroissement journalier, dont la valeur pour un cultivar donné est principalement dépendante de l'âge reproducteur des individus de $S$. avenae, semble être l'élément le plus discriminant entre cultivars très ou peu favorables à ce puceron.

Une analyse de variance portant sur les valeurs de $r_{m}$ obtenues sur les mêmes répétitions révèle des différences très significatives au seuil 1 p. 100 entre les cultivars étudiés $\left(\mathrm{F}_{247}^{24}=2,749\right)$. Les résultats de la comparaison multiple des moyennes par le test de DUNCAN au seuil 1 p. 100 sont présentés dans la dernière colonne du tableau 3 : ils peuvent être résumés ainsi :

- les cultivars «Vala », «Cajka » et « Mironovskaïa 808 "sont peu favorables, à ce stade, pour $S$. avenae $\left(\mathrm{r}_{\mathrm{m}}\right.$ significativement plus faible) par rapport à «PI 94585 », «Cadet », «Champlein », « Iljit- 


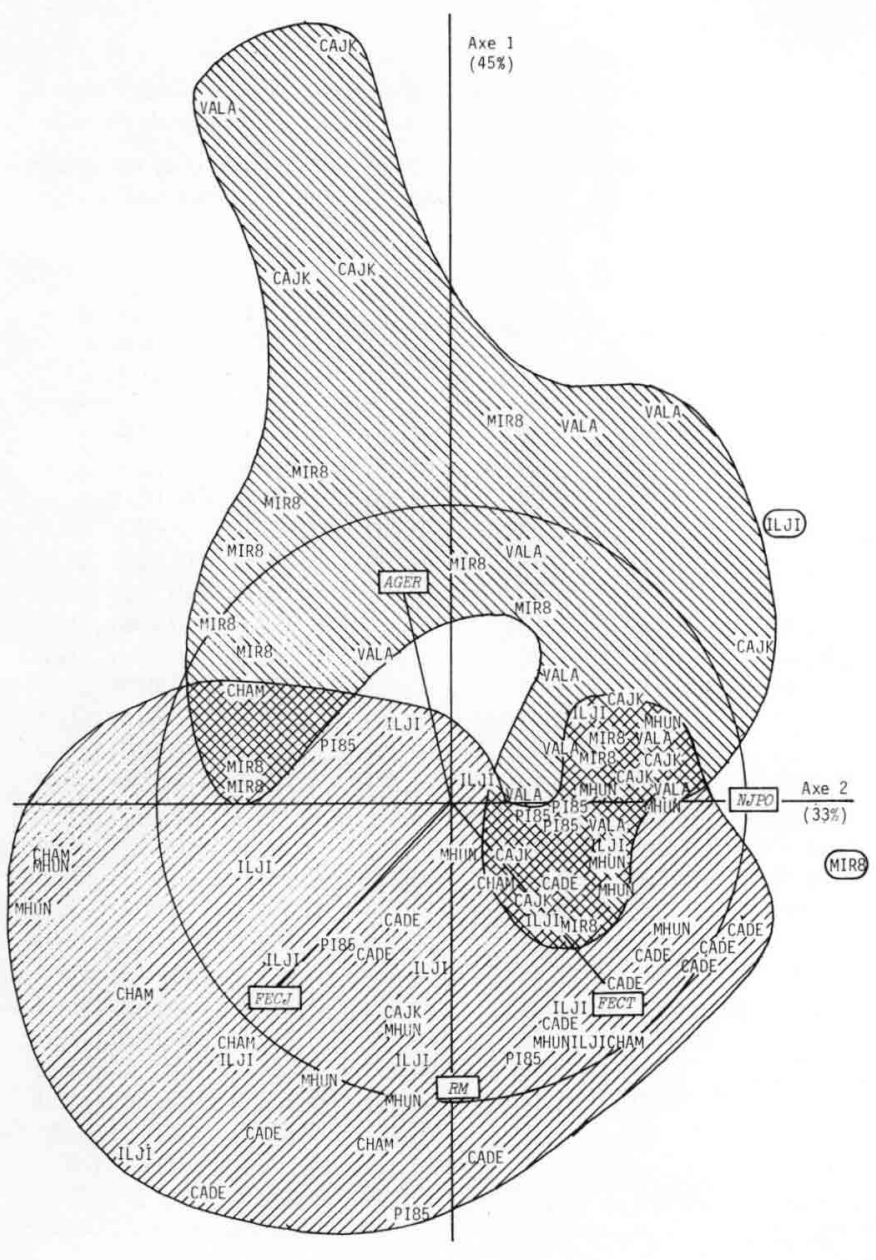

chevka » et «Maris Huntsman », au contraire très favorables ;

- le cultivar "Vala " est moins favorable que « Fidel » et « Nongda 141 »;

— «Cajka » est moins favorable que « Fidel »;

- les autres cultivars sont intermédiaires.

Ces résultats sont corroborés par ceux de l'A.C.P.

Figure 1

Plan factoriel d'une analyse des correspondances effectuée sur l'ensemble des cultivars et des répétitions et portant sur 5 variables mesurées au stade "plantule non vernalisée ".

AGER âge au moment du début de la reproduction (en jours), NJPO nombre de jours de ponte,

FECT fécondité totale (en nombre de larves),

FECI fécondité moyenne journalière (en nombre de larves par femelle et par jour),

RM taux intrinsèque d'accroissement journalier.

Seuls sont représentés les individus appartenant aux cultivars les plus remarquables. Ceux-ci sont figurés par leur abréviation indiquée au tableau 2 ou au $\S I I$.A.

Factorial plan of a correspondence analysis on all cultivars and replicates, with 5 variables estimated at the "non-vernalized plant " stage.

AGER aphid age at the beginning of reproduction (in days),

NJPO number of days of laying,

FECT total fecundity (in number of larvae),

FECJ mean daily fecundity (in number of larvae per female per day),

RM intrinsic rate of increase.

Only individuals belonging to the more noteworthy cultivars are represented, indicated by their abbreviation (see table 2 and $\$$ II.A).

TABLEAU 4

Caractéristiques démographiques de $\mathrm{S}$. avenae sur 24 cultivars de blé d'hiver au stade épiaison-maturité laiteuse. Les écarts-types des valeurs moyennes sont indiqués entre parenthèses. Les $r_{m}$ avec les mêmes lettres ne sont pas significativement différents au seuil 1 \% du test de DuNCAN. Demographic characteristics of S. avenae on 24 cultivars of winter wheat at the heading-milky ripeness stage. Standard deviations of the means are between brackets. The $r_{m}$ with a common letter did not differ significantly at the l \% threshold of DUNCAN's range test.

\begin{tabular}{|c|c|c|c|c|c|c|c|c|c|c|c|}
\hline \multirow{2}{*}{$\begin{array}{l}\text { Cultivars } \\
\text { MHUN }\end{array}$} & \multirow{2}{*}{$\begin{array}{l}\text { (1) } \\
09\end{array}$} & \multicolumn{2}{|c|}{$\begin{array}{l}\text { Age reproducteur } \\
\text { (jour) }\end{array}$} & \multicolumn{2}{|c|}{$\begin{array}{l}\text { Nombre de jours } \\
\text { de ponte }\end{array}$} & \multicolumn{2}{|c|}{$\begin{array}{l}\text { Fécondité totale } \\
\text { (Larves/Q) }\end{array}$} & \multicolumn{2}{|c|}{$\begin{array}{l}\text { Fécondité journalière } \\
\text { (Larves/q/jour) }\end{array}$} & \multicolumn{2}{|c|}{$\begin{array}{l}\text { Taux intrinsèque } \\
\text { d'accroissement } \\
\text { journalier } r_{m}\end{array}$} \\
\hline & & 7,00 & $(0,71)$ & 15,11 & $(2,89)$ & 68,67 & $(11,74)$ & 4,62 & $(0,79)$ & 0,3439 & $(0,024)$ a \\
\hline CHAM & 10 & 7,20 & $(0,79)$ & 17,10 & $(3,14)$ & 74,10 & $(04,82)$ & 4,46 & $(0,82)$ & 0,3347 & $(0,019)\{a b$ \\
\hline ROAZ & 08 & 7,75 & $(1,17)$ & 17,75 & $(3,96)$ & 73,50 & $(08,72)$ & 4,33 & $(1,07)$ & 0,3275 & $(0,052)\{\mathrm{ac}$ \\
\hline CADE & 08 & 8,13 & $(0,35)$ & 18,13 & $(3,00)$ & 80,13 & $(03,09)$ & 4,54 & $(0,82)$ & 0,3225 & $(0,020)$ ad \\
\hline CAPI & 09 & 8,00 & $(0,71)$ & 18,11 & $(4,78)$ & 78,22 & $(06,30)$ & 4,52 & $(0,97)$ & 0,3189 & $(0,020)\{\mathrm{ad}$ \\
\hline MIR8 & 09 & 7,78 & $(0,67)$ & 18,56 & $(1,67)$ & 69,67 & $(07,31)$ & 3,76 & $(0,36)$ & 0,3147 & $(0,027)$ ae \\
\hline KIAN & 08 & 8,25 & $(0,46)$ & 19,50 & $(1,20)$ & 78,88 & $(03,98)$ & 4,05 & $(0,26)$ & 0,3123 & $(0,012)\{\mathrm{af}$ \\
\hline CAM7 & 10 & 7,90 & $(0,74)$ & 17,00 & $(3,09)$ & 73,10 & $(09,21)$ & 4,37 & $(0,65)$ & 0,3121 & $(0,026)$ af \\
\hline FIDE & 10 & 7,60 & $(1,08)$ & 21,70 & $(4,67)$ & 70,50 & $(13,78)$ & 3,31 & $(0,73)$ & 0,3119 & $(0,038)\{$ af \\
\hline ACHT & 03 & 8,00 & $(0,00)$ & 17,00 & $(1,00)$ & 80,67 & $(00,58)$ & 4,76 & $(0,28)$ & 0,3084 & $(0,010)$ be \\
\hline CAM3 & 09 & 8,11 & $(0,60)$ & 19,22 & $(2,54)$ & 72,78 & $(04,89)$ & 3,73 & $(0,37)$ & 0,3080 & $(0,019)$ be \\
\hline PI85 & 07 & 8,71 & $(0,76)$ & 18,57 & $(0,98)$ & 71,00 & $(01,83)$ & 3,83 & $(0,23)$ & 0,3064 & $(0,019)$ be \\
\hline MIXB & 04 & 8,75 & $(0,50)$ & 15,00 & $(0,00)$ & 72,75 & $(01,50)$ & 4,85 & $(0,10)$ & 0,3035 & $(0,015)\{\mathrm{bh}$ \\
\hline BRHB & 06 & 8,17 & $(0,41)$ & 22,83 & $(2,93)$ & 65,00 & $(11,63)$ & 2,85 & $(0,40)$ & 0,3018 & $(0,014)\{\mathrm{bi}$ \\
\hline MI25 & 07 & 8,29 & $(0,49)$ & 18,14 & $(4,02)$ & 66,57 & $(10,34)$ & 3,83 & $(0,90)$ & 0,2998 & $(0,021)$ (ci \\
\hline MIXO & 08 & 8,38 & $(0,52)$ & 16,63 & $(3,16)$ & 66,75 & $(08,48)$ & 4,09 & $(0,65)$ & 0,2997 & $(0,013)$ (ci \\
\hline NIZK & 09 & 8,67 & $(0,71)$ & 17,78 & $(2,99)$ & 72,22 & $(06,08)$ & 4,19 & $(0,92)$ & 0,2941 & $(0,015)\{\mathrm{cj}$ \\
\hline ND41 & 11 & 8,91 & $(0,54)$ & 19,64 & $(1,36)$ & 68,36 & $(07,70)$ & 3,49 & $(0,35)$ & 0,2903 & $(0,014)\{\mathrm{dj}$ \\
\hline CAJK & 12 & 8,83 & $(0,72)$ & 21,08 & $(3,29)$ & 69,33 & $(08,47)$ & 3,36 & $(0,65)$ & 0,2823 & $(0,009)$ \{ej \\
\hline VALA & 10 & 8,40 & $(0,52)$ & 21,10 & $(0,88)$ & 66,20 & $(10,00)$ & 3,14 & $(0,48)$ & 0,2791 & $(0,021)(\mathrm{fj}$ \\
\hline ILJI & 11 & 9,00 & $(0,63)$ & 18,82 & $(1,33)$ & 71,36 & $(07,06)$ & 3,81 & $(0,48)$ & 0,2767 & $(0,015)$ lgj \\
\hline ND40 & 08 & 8,38 & $(0,52)$ & 25,00 & $(2,27)$ & 60,38 & $(08,43)$ & 2,42 & $(0,25)$ & 0,2728 & $(0,005)\{h j$ \\
\hline JUBI & 06 & 8,83 & $(0,75)$ & 22,33 & $(2,42)$ & 60,50 & $(13,98)$ & 2,76 & $(0,80)$ & 0,2695 & $(0,023)$ (ij \\
\hline PI71 & 08 & 8,25 & $(0,46)$ & 19,88 & $(6,17)$ & 50,88 & $(15,63)$ & 2,60 & $(0,34)$ & 0,2659 & $(0,017) \mathrm{j}$ \\
\hline
\end{tabular}

(1) Nombre de répétitions. 
précédente et illustrés par la figure 1 ; on y a représenté les nuages des points-individus appartenant aux groupes de cultivars peu ou très favorables au puceron : on note un faible recoupement de ces 2 ensembles. On remarque, de plus, que pour les cultivars favorables, il existe une variabilité relativement faible des valeurs de $r_{m}$ et/ou de l'âge reproducteur (étalement du nuage des individus dans le sens de l'axe 2) ; par contre, pour les cultivars peu favorables, et particulièrement pour "Cajka », il existe une grande variabilité de ces valeurs individuelles, illustrée par l'étalement du nuage dans le sens de l'axe 1 .

\section{B. Les essais au stade épiaison - maturité laiteuse}

Les valeurs moyennes des paramètres estimées à ce stade sur $S$. avenae et concernant seulement 24 cultivars sont présentées dans le tableau 4. Les mêmes analyses statistiques ont été effectuées.

L'interprétation d'une A.C.P. effectuée sur l'ensemble des individus (200) pour les 24 cultivars et les variables déjà citées est sensiblement la même que précédemment.

Le pourcentage de l'inertie totale représenté par les 2 premières composantes est de $78 \mathrm{p}$. 100 : la $1^{\text {re }}$ composante (54 p. 100 de l'inertie) est fortement corrélée à RM et FECJ (coefficients respectifs : 0,804 et 0,791 ) et la $2^{\mathrm{e}}$ (24 p. 100 de l'inertie) à NJPO principalement (coefficient : 0,560 ).

On note encore une corrélation remarquable entre RM et AGER ainsi qu'entre RM et FECJ (coefficients respectifs : $-0,75$ et 0,63 ).

L'interprétation des axes du plan factoriel (fig. 2) est donc la même qu'au stade plantule : le $1^{\text {er }}$ axe oppose les cultivars sur lesquels $S$. avenae se multiplie rapidement, du fait d'un $r_{m}$ élevé associé à une forte fécondité journalière, à ceux sur lesquels il se multiplie lentement. De même, l'axe 2 oppose plutôt les individus à longue ou brève durée de ponte.

Une analyse de variance effectuée sur les valeurs des $\mathrm{r}_{\mathrm{m}}$ obtenues révèle des différences très significatives au seuil 1 p. 100 entre les cultivars $\left(F_{176}^{23}=7,587\right)$. Le test de comparaisons multiples des moyennes de DUNCAN au seuil 1 p. 100 donne les résultats présentés dans le tableau 4 et résumés ainsi :

- les cultivars «PI $94571 »$, « Jubilejna », « Nongda 140 », «Iljitchevka », «Vala » et «Cajka » sont peu favorables à $S$. avenae, comparés aux cultivars " Maris Huntsman », « Champlein », « Roazon», «Cadet » et «Capitole » plutôt très favorables à ce puceron,

- les autres cultivars sont intermédiaires.

La figure 2 présente une illustration de l'A.C.P., simplifiée aux 2 groupes différenciés par l'analyse de variance : les nuages des points individus appartenant à ces 2 groupes sont bien distincts, à un individu près (sur « Roazon »), ce qui corrobore les résultats précédents.

\section{Comparaison des résultats obtenus aux stades plantule non vernalisée et épiaison-maturité lai- teuse}

Sur l'ensemble des cultivars, on observe globalement une augmentation de la valeur moyenne du $r_{m}$

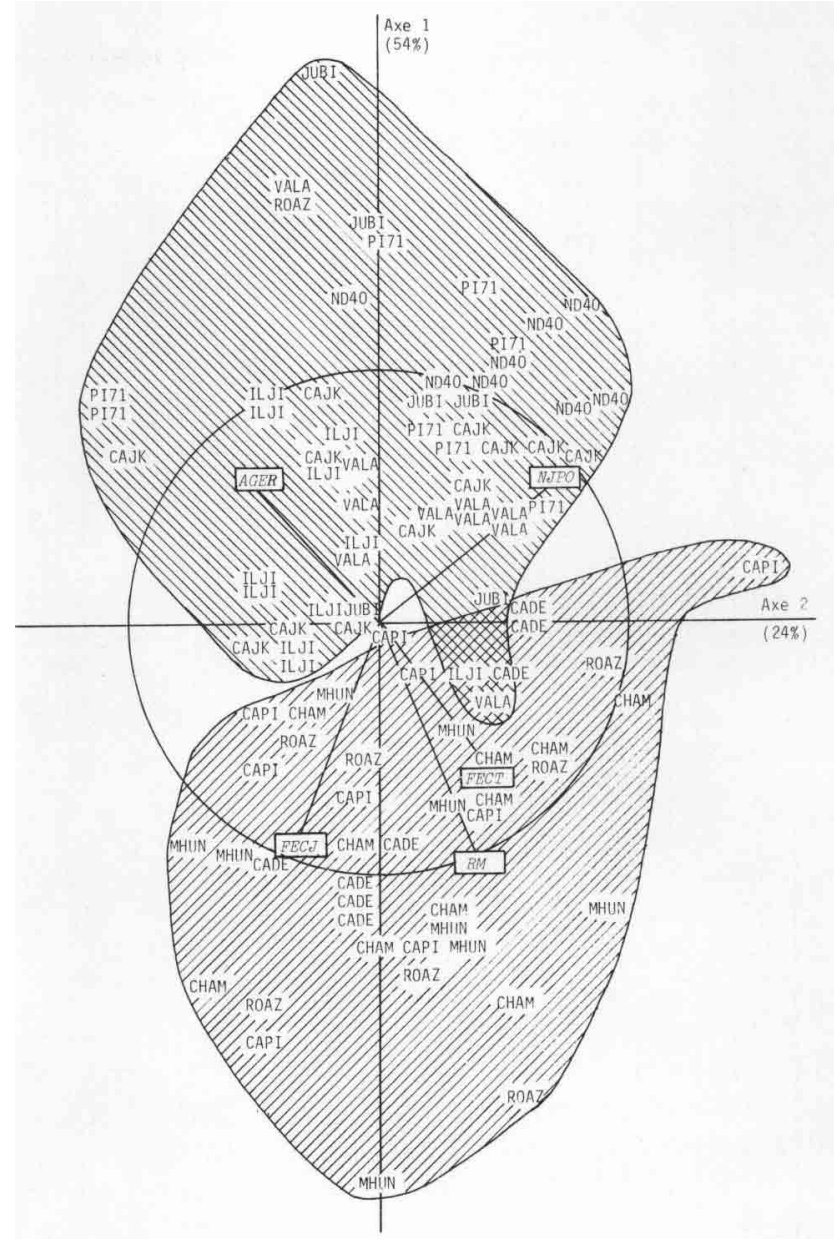

Figure 2

Même légende que figure 1 pour les 5 variables mesurées au stade «épiaison-maturité laiteuse ».

Same legend as figure 1 for 5 variables estimated at the " headingmilky ripeness " growth stage.

entre le stade plantule $\left(r_{m}=0,2756\right)$ et le stade épiaison $\left(r_{m}=0,3022\right)$, déterminée principalement par un raccourcissement de la durée de préreproduction (respectivement $8,7 \mathrm{j}$ et $8,2 \mathrm{j}$ ) et un accroissement de la fécondité journalière moyenne de $S$. avenae $(2,4$ et $3,8 \mathrm{~L} / Q / \mathrm{j})$. Cependant, la comparaison par le test « $\mathrm{t}$ » de DUNCAN des $r_{\mathrm{m}}$ moyens entre stades phénologiques permet de distinguer 2 tendances chez les cultivars (fig. 3) :

- pour tous les cultivars très favorables au stade épiaison comme pour 9 des 13 cultivars jugés intermédiaires et pour «Cajka » et «Vala » défavorables, le $\mathrm{r}_{\mathrm{m}}$ obtenu à ce stade est significativement supérieur à celui obtenu au stade plantule, conformément à la tendance générale (fig. 3a) ;

- il n'y a, au contraire, pas de différence significative entre le stade plantule et le stade épiaison pour 4 des cultivars intermédiaires au stade épiaison («Fidel », «PI $94585 »$, « Mironovskaila 808 » et «Nongda $141 »)$ comme pour les 4 cultivars les plus défavorables à ce stade (fig. $3 b$ ).

En conséquence, le caractère favorable ou défavorable des différents cultivars à la multiplication de $S$. avenae n'est pas constant dans tous les cas aux 

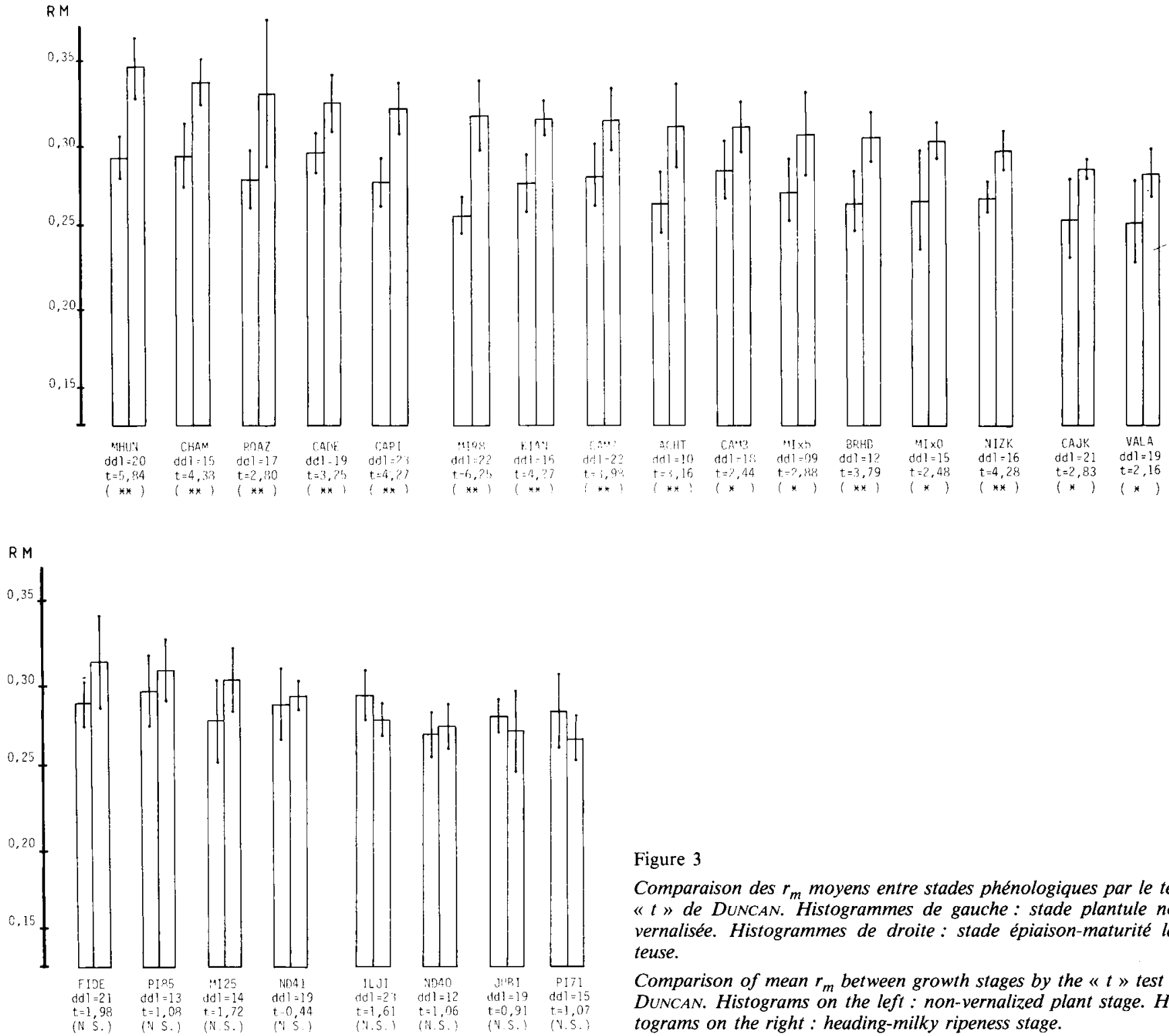

Figure 3

Comparaison des $r_{m}$ moyens entre stades phénologiques par le test « $t$ " de DunCaN. Histogrammes de gauche: stade plantule non vernalisée. Histogrammes de droite: stade épiaison-maturité laiteuse.

Comparison of mean $r_{m}$ between growth stages by the « $t$ 》 test of DUNCAN. Histograms on the left: non-vernalized plant stage. Histograms on the right : heading-milky ripeness stage.

2 stades phénologiques étudiés, comme le montre la figure 4. Notamment :

- «Champlein », « Maris Huntsman » et « Cadet» restent toujours fortement multiplicateurs,

- «Cajka » et «Vala », au contraire, manifestent toujours une certaine résistance à $S$. avenae,

- "Iljitchevka " est remarquable dans la mesure où, très favorable au stade plantule, il est très défavorable à l'épiaison,

- les cultivars «PI 94571 », «Jubilejna » et « Nongda 140 » sont aussi remarquables du fait qu'ils semblent acquérir à l'épiaison (ou à un stade intermédiaire) un caractère de résistance relative très net à S. avenae,

- enfin, "Mironovskaïa 808 », nettement résistant au stade plantule, est intermédiaire, voire favorable à ce puceron au stade épiaison.

On remarque de plus une relative similitude, dans le « comportement " par rapport à ce phénomène, de cultivars proches d'un point de vue génétique comme « Mironovskaïa 808 », « $(\mathrm{Mi} \times$ b) 5.4.4.3.2.", " Mironovskaïa 25 », " Mironovskaïa $\times$ Ouloutchen- naïa » et "Mironovskaïa $\times$ Nizkorostla », comme «Campénéac 13 » et "Campénéac 17 » et enfin « Nongda 140 » et « Nongda 141 » (fig. 4).

\section{DISCUSSION ET CONCLUSION}

La méthode de "sélection » sur le comportement au champ des cultivars s'est révélée d'une grande fiabilité en tant que critère de choix du matériel végétal et ceci, malgré les faibles populations de $S$. avenae développées au Rheu en 1983. Effectué au stade épiaison-maturité laiteuse de la majorité des cultivars de la collection, le choix de cultivars peu favorables à $S$. avenae est confirmé par nos expériences en conditions contrôlées à ce même stade phénologique. Tous ceux ainsi sélectionnés, à l'exception de "Cadet", présentent un $r_{m}$ inférieur à celui de "Capitole", seule variété étudiée antérieurement et présente dans cette collection. Cette méthode de prospection rapide pourrait être étendue avec intérêt à d'autres collections existantes et révéler des différences plus élevées 


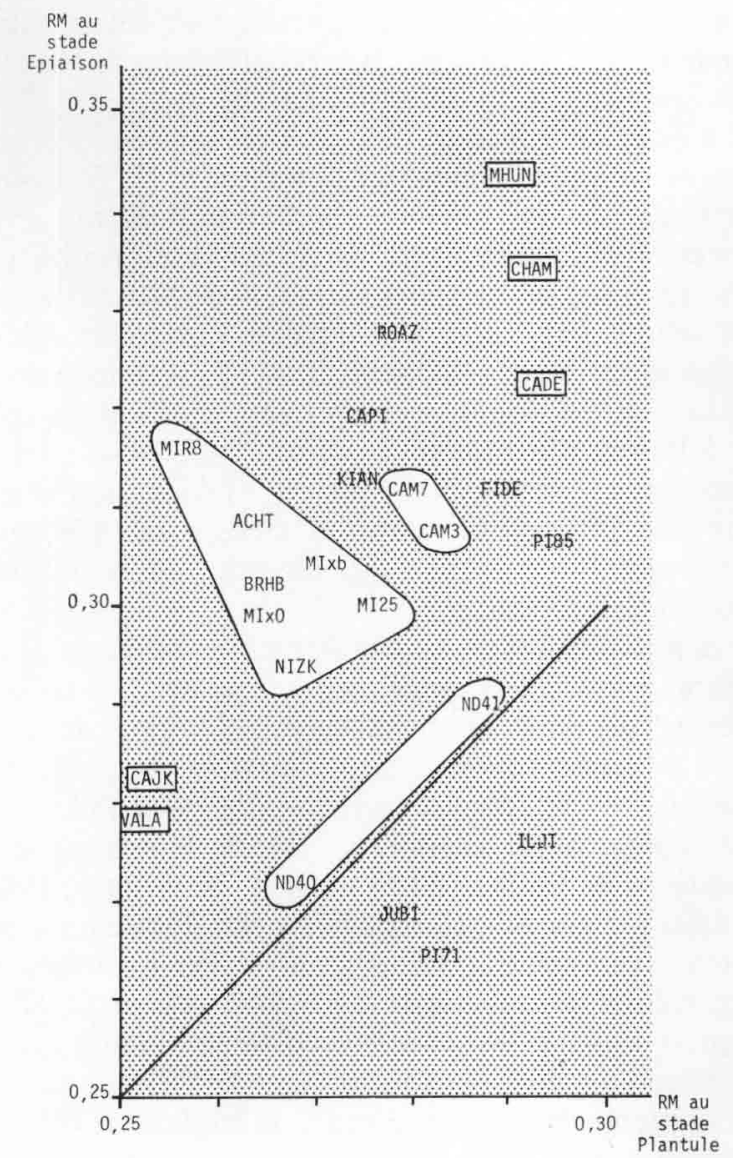

Figure 4

Comparaison des $r_{m}$ aux stades plantule non vernalisée et épiaisonmaturité laiteuse pour chaque cultivar étudié.

Comparison of $r_{m}$ values for non-vernalized plant and headingmilky ripeness stages, for each cultivar.

d'infestations en cas de plus fortes populations de S. avenae.

La détermination du taux intrinsèque d'accroissement journalier pour caractériser la résistance par antibiosis des cultivars à $S$. avenae présente un grand intérêt :

- d'un point de vue statistique, cette étude montre que c'est le paramètre le plus discriminant pour séparer les cultivars fortement multiplicateurs de $S$. avenae de ceux qui le sont peu ;

- d'un point de vue biologique, sa détermination permet de prendre en compte les effets relatifs d'antibiosis que peut manifester le cultivar-hôte en agissant sur les différents paramètres d'accroissement des populations du puceron. Dans des expériences analogues concernant la résistance de cultivars et espèces du genre Vicia à Aphis fabae (Scop.) et Acyrthosiphon pisum (Harris), BIRCH \& HOLT (1981) notent pareillement que la mesure de chacun des paramètres dynamiques (fécondité, durée de développement ou longévité) ne suffit généralement pas à une prévision de la croissance des populations sur ces hôtes. En particulier, la faible fécondité des pucerons observée sur certains cultivars de l'espèce Vicia faba L. est compensée par une courte durée de développement. De ce fait, le taux d'accroissement des populations mesuré par $r_{m}$ n'est pas aussi faible que le laissent prévoir les seules mesures de la fécondité. Ils constatent, au contraire, que les différences de $\mathrm{r}_{\mathrm{m}}$ entre cultivars de $V$. faba sont similaires à celles qui concernent la durée de développement des pucerons. Nous avons de même signalé la corrélation remarquable qui lie, dans notre cas, le paramètre $r_{m}$ à la durée de préreproduction de $S$. avenae pour les différents cultivars étudiés ;

- d'un point de vue pratique, il découle que l'évaluation rapide et facile de ce dernier paramètre pourrait constituer une étape préliminaire pour la sélection de cultivars particulièrement intéressants, pour lesquels serait ensuite déterminé $r_{m}$. On étudie actuellement les possibilités de simplification des expérimentations, longues et fastidieuses (entre 20 et $30 \mathrm{j}$ d'observations quotidiennes) normalement nécessaires à la détermination de ce paramètre. La fécondité journalière pourrait être notée seulement sur une période égale à la durée de préreproduction (ces 2 périodes cumulées contribuent d'après DE LOACH (1974) à 95 p. 100 de la valeur du $\mathrm{r}_{\mathrm{m}}$ ) et avec un pas d'observations de 2 à $4 \mathrm{j}$ (BARON, comm. pers.).

Les expérimentations ont été menées à 2 stades phénologiques :

- le stade plantule non vernalisée est uniquement intéressant d'un point de vue expérimental dans la mesure où il est rapide à obtenir en grande quantité à partir de semences des cultivars à comparer. Les observations sont aisées étant donné le faible volume foliaire développé :

- le stade épiaison-maturité laiteuse présente un intérêt de vérification pratique dans la mesure où, dès leur émergence, les épis sont, dans la nature, rapidement colonisés par $S$. avenae qui y atteint la phase culminante de ses effectifs. C'est aussi à ce stade phénologique que les dégâts quantitatifs et qualitatifs provoqués par ce puceron sont les plus importants (VEREIJKEN, 1979). Cependant, l'obtention du matériel végétal à ce stade est longue et les expérimentations sont encombrantes et moins faciles à réaliser que sur plantules.

Il semble que les tests sur plantules ne permettent pas de sélectionner un ensemble de cultivars dont on soit sûr qu'ils soient " résistants " à $S$. avenae à tous les stades de leur évolution phénologique, ce qui n'est le cas ici que pour «Cajka " et "Vala ». Dans le cas de "Mironovskaïa 808 ", la résistance décelée au stade plantule disparaît au stade épiaison. Au contraire pour «Iljitchevka », la résistance remarquable mise en évidence à l'épiaison et dont la sélection peut être intéressante, n'apparaît pas au stade plantule.

Cependant, dans les autres cas que nous avons observés, le classement des cultivars en fonction de leur résistance relative au stade plantule non vernalisée pourrait être extrapolé à l'ensemble des stades qui précèdent l'épiaison.

En conclusion, sur l'ensemble des cultivars, l'augmentation de la valeur moyenne de $r_{m}$ entre les 2 stades phénologiques est conforme aux résultats de WATT (1979) obtenus dans les mêmes conditions expérimentales sur avoine (cultivar "Aster ») et suggérés par des observations au champ sur le cultivar « Maris Huntsman ». De même VEREIJKEN (1979) considère, d'une manière générale, les stades allant de l'anthèse à la maturité laiteuse comme les plus favorables à 
S. avenae. Parmi les cultivars ayant fait l'objet d'observations antérieures (DEDRYVER \& DI PIETRO, 1986), “ Maris Huntsman » confirme le fort caractère multiplicateur pour $S$. avenae constaté en conditions naturelles. "Champlein », très favorable en conditions contrôlées ne s'était pas distingué au champ, peut-être à cause de sa relative précocité. "Roazon" est aussi très favorable à $S$. avenae en conditions contrôlées : l'hypothèse de sa "résistance apparente » au champ, due à sa sensibilité aux maladies foliaires s'en trouve renforcée. « Fidel » et « Mironovskaïa 808 » manifestaient en conditions naturelles un caractère relativement peu favorable à $S$. avenae que l'on retrouve ici, particulièrement pour le second au stade plantule non vernalisée.

Parmi les nouveaux cultivars expérimentés, seul «Cadet » se révèle d'une « sensibilité » comparable à celle de «Maris Huntsman » et "Champlein ». Pour l'ensemble des autres cultivars, on note, au moins à l'un des 2 stades phénologiques, un caractère de résistance marqué par rapport à ces 2 variétés. Les plus remarquables sont "Cajka » et «Vala » et au stade épiaison «Iljitchevka». Les différences de taux d'accroissement entre des cultivars comme « Maris Huntsman » $\left(r_{m}=0,35\right)$ et «PI $571 »\left(r_{m}=0,27\right)$ ont sans aucun doute une signification dans l'hypothèse plausible d'une phase d'accroissement exponentiel des populations de $S$. avenae au champ à partir de l'épiaison. En $21 \mathrm{j}$, durée moyenne de la période allant de l'épiaison à la maturité laiteuse du blé, 1 virginipare aptère de $S$. avenae pourrait donner naissance à 1560 individus dans le $1^{\text {er }}$ cas et à 290 dans le $2^{\mathrm{e}}$ cas, en l'absence de facteurs limitants. Le rapport des populations développées durant cette période serait ainsi de 5 pour 1 entre un cultivar fortement multiplicateur et un autre qui le serait peu.

L'intérêt d'une intégration de tels degrés de résistance partielle dans de nouvelles variétés de blé est suggéré par l'utilisation d'un modèle de simulation des populations de $S$. avenae sur céréales par CARTER \& DiXON (1981). Ces auteurs montrent notamment que d'assez faibles diminutions des taux de survie et de reproduction du puceron peuvent conduire à une importante réduction des densités de $S$. avenae au moment du maximum des populations de ce ravageur. ACREMAN (1984) en déduit qu'une résistance partielle comparable à celle que nous avons mise en évidence suffirait à remplacer les traitements insecticides systématiques actuellement appliqués en Grande-Bretagne contre ce puceron. Pour nos cultivars les plus remarquables, il semble néanmoins nécessaire de vérifier, pour d'autres souches de $S$. avenae et en conditions naturelles, l'expression du caractère faiblement multiplicateur déjà suggéré par nos observations antérieures et les résultats de la sélection au champ.

La résistance par antibiosis peut affecter certains paramètres dynamiques des populations de $S$. avenae que nous n'avons pas pris en compte dans nos expériences. Notamment, une partie de la mortalité précoce observée sur certains individus de $S$. avenae peut en être une manifestation, de même l'orientation morphogénique du développement de $S$. avenae vers la production de virginipares ailés peut varier d'un cultivar à l'autre (DI PIETRO \& DEDRYVER, 1984) : l'aptitude d'un cultivar à favoriser cette production d'ailés peut être intéressante dans la mesure où elle serait défavorable au maintien de colonies denses de pucerons sur ce cultivar.

Les mécanismes physiques et/ou biochimiques de tels phénomènes de résistance sont à élucider. Ils ne semblent pas liés à des caractères morphologiques des cultivars concernés, comme l'aristation des épis, cause supposée du caractère moins favorable de "Fidel 》 au stade épiaison par rapport à " Maris Huntsman » en conditions naturelles (DEDRYVER \& DI PIETRO, 1986). En effet «Vala », « Iljitchevka » et « Jubilejna » présentent une aristation faible à nulle. Une hypothèse est suggérée par les travaux de ARGANDOÑA et $a l$. (1980a et b) qui ont mis en évidence une corrélation entre la concentration d'acides hydroxamiques (DIMBOA) dans de jeunes plants de cultivars d'orge, de seigle et de blé (Triticum durum L. et $T$. aestivum L.) et le taux de multiplication de population de $M$. dirhodum. Cette concentration varie aussi en fonction des différents organes et de leur âge : chez $T$. durum, elle est plus importante dans les jeunes feuilles que dans les feuilles plus âgées et nulle dans les graines. Cette hypothèse plausible d'une résistance par antibiosis liée à des caractères biochimiques variables entre cultivars ou d'un stade phénologique à l'autre mérite d'être approfondie dans le cas qui nous intéresse, notamment dans la mesure où elle pourrait aboutir à la mise en place de tests de sélection simples, rapides et facilement reproductibles.

Reçu le 30 juillet 1985. Accepté le 24 janvier 1986.

\section{REMERCIEMENTS}

Les auteurs remercient vivement G. Doussinault de la Station d'Amélioration des Plantes (I.N.R.A., Le Rheu) pour l'aide qu'il leur a apportée et S. D. WRATTEN (Département de Biologie de l'Université de Southampton, Grande-Bretagne) pour la communication du programme en ALGOL 68 de détermination du $r_{m}$.

\section{RÉFÉRENCES BIBLIOGRAPHIQUES}

Acreman T. M., 1984. The contribution of resistance to cereal aphid control. Proc. 1984 British crop Protection Conference, Pests and Diseases, 1, 31-36.

Argandoña V. H., Luza J. G., Niemeyer H. M., Corcuera L. J., 1980a. Role of hydroxamic acids in the resistance of cereals to aphids. Phytochemistry, 19, 1665-1668.
Argandoña V. H., Niemeyer H. M., Corcuera L. J., 1980b. Content and distribution of hydroxamic acids in wheat. Plant Physiol., 65 (6), 758-761.

Birch L. C., 1948. The intrinsic rate of natural increase of an insect population. J. an. Ecol., 17, 15-26. 
Birch N., Holt J., 1981. Aphid resistance in Vicia in relation to non-protein amino acids. Bull. SROP, IV (1), 133-139.

Carter N., Dixon A. F. G., 1981. The use of insect population simulation models in breeding for resistance. Bull. SROP, IV (1), 21-24.

De I.oach C. J.. 1974. Rate of increase of popula!ions of cabbage. green peach and turnip aphids at constant temperatures. Ann. entomol., Soc. Am., 67, 332-361.

Dedryver C. A., Di Pietro J. P., 1984. Comparaison de l'évolution au champ des populations de pucerons des céréales sur différents cultivars de blé d'hiver. Bull. SROP, VII (4), 9-10.

Dedryver C. A., Di Pietro J. P., 1986. Biologie des pucerons des céréales dans l'ouest de la France: VI - Etude comparative des fluctuations au champ de Sitobion avenae F., Metopolophium dirhodum Wlk, et Rhopalosiphum padi L. sur différents cultivars de blé d'hiver. Agronomie, 6 (1), 75-84

Di Pietro J. P., Dedryver C. A., 1984. Quelques facteurs conditionnan! l'expression du potentiel biotique des pucerons des céréales sur différents cultivars de blé d'hiver. Bull. SROP, VII (4), 11-14.

Hinz B., Daebeler F., 1974. Untersuchungen zur Anfälligkeit verschiedener Getreidearten und -sorten gegenüber Getreideblattläusen. Arch. Phytopathol. Pflanzensch., 10 (5), 341-346.

Hinz B., Daebeler F., 1976. Zur Beeinflussung der Er1ragsbildung bei Winter weizensorten durch die grosse Getreideblattlaus Macrosiphum (Sitobion) avenae F. Arch. Phytopathol., Pflanzensch., 12 (2), 111-116.

Kogan M., Ortman E. F., 1978. Antixenosis a new term proposed to define Painter's « non-preference " modality of resistance. Bull. Soc. Am., 24, 175-176.
Lebart L., Morineau A., Fenelon J. P., 1979. Traitement des données statistiques Ed. Bordas, Paris, 510 p.

Lowe H. J. B., 1980. Resistance to aphids in immature wheat and barley. Ann. appl., Biol., 95, 129-135.

Lowe H. J. B., 1981. Resistance and susceptibility to colour forms of the aphid Sitobion avenae in spring and winter wheats (Triticum aestivum). Ann. appl. Biol., 99, 87-88.

Iowe H. J. B., 1982. Some observations on susceptibility and resistance of winter wheat to the aphid Sitobion avenae (F.) in Britain. Crop Prot., 1, 431-440.

Painter R. H., 1951. Insect resistance in crop plants. Macmillan, New York, 520 p.

Painter R. H., 1958. Resistance of plants to insects. Annu. Rev. Entomol., 3, 267-290.

Sotherton N. W., Van Emden H. F., 1982. Laboratory assessments of resistance to the aphids Sitobion avenae and Metopolophium dirhodum in three Triticum species and two modern wheat cultivars. Ann. appl. Biol., 101, 99-107.

Vereijken P. H., 1979. Feeding and multiplication of three cereal aphid species and their effect on yield of winter wheat. Agric. Res. Rep. (Verslagen van landbouwkundige onderzoekingen), 888, 1-58.

Wall A. D., 1979. The effects of cereal growth stages on the reproductive activity of Sitobion avenae and Metopolophium dirhodum. Ann. appl. Biol., 91, 147-157.

Zadoks J. C., Chang T. T., Konzak C. F., 1977. Un code décimal pour les stades de croissance des céréales. Phytiatr. Phytopharm., 26, $129-140$ 